\title{
The ACMG CYTO2000 subcommittee?
}

Although Lejeune discovered that trisomy 21 was the cause of Down Syndrome almost 40 years ago and began the association of clinical findings with cytogenetics, ${ }^{1}$ significant knowledge gaps in clinical cytogenetics still exist. In addition, because of the increasing emphasis on cost efficiency driven by today's health care systems, many cytogeneticists have begun to wonder whether some of the cytogenetic guidelines recommended by the American College of Medical Genetics (ACMG) can be improved. Some of us realized that to truly understand some of the issues associated with clinical cytogenetics, one would need to collect and analyze data from many laboratories. To foster such interlaboratory cooperation, we began the CYTO2000 Working Group in October 1995. Its purpose is to serve as a model for the investigation of the practice and science of cytogenetics. The steering committee of the working group later was formalized as a Subcommittee of the ACMG Quality Assurance Subcommittee. The first task of the CYTO2000 Subcommittee is to collect data from participating laboratories so that laboratory standards will be based on empiric data, instead of rule-of-thumb estimates.

The CYTO2000 process has the following guiding principles: 1) participation by laboratories is dictated by common interests; 2) priorities are set by participants; 3 ) studies to address specific questions are determined by consensus; 4 ) studies have strict deadlines for participants; 5) data remain confidential; 6) the Subcommittee ensures that information is disseminated and analyzed; and 7) results and interpretations represent consensus opinion.

The Subcommittee aids in the formation of working groups that pursue various projects. A working group is composed of the CYTO2000 Subcommittee (which provides leadership for working group projects as well as logistical and statistical support) and members (or participants) in working group projects. Group members may change from project to project. CYTO2000 projects arise from discussions at open meetings that have been scheduled at the ACMG and American Society of Human Genetics (ASHG) meetings since 1996. Project leaders are volunteers responsible for developing data collection instruments designed to answer specific clinical research questions. The Subcommittee helps distribute those instruments to potential participants, collect the data from participating laboratories, and oversee the analysis of those data and the reporting of findings.

Projects are initiated only if proposal generates sufficient interest among potential participants and has at least one volunteer to lead the project. For example, initial participants of the working group agreed that the first study should address whether the 20 -cell or 15-colony count recommended in the ACMG guidelines for the amniotic fluid cell culture is appropriate. Although this issue could be addressed by a prospective study that would involve counting as many as 50 cells in amniotic fluid cultures, this approach was tabled because laboratory directors said that they could not devote resources to routinely count that many cells. Instead, the working group decided to address the same question retrospectively, using existing laboratory data. The study was piloted with eight laboratories and later expanded to 26 laboratories. The results of the study will be submitted to this journal.

Having shown that the process works, we decided to initiate two additional studies: the CYTO2000 Blood Mosaicism Study will be similar to the amniotic fluid mosaicism study, and the Amniotic Fluid Mosaicism Outcome Study will examine the clinical outcomes in cases of prenatally detected mosaicism. Both studies are currently in the planning stage.

To inform, disseminate, and communicate with participants (current and future), the CYTO2000 subcommittee has created a World Wide Web page as a part of the ACMG web site. The web page address (URL) of the CYTO2000 Group is: http://www.faseb.org/genetics/acmg/ cyto2000/cytomain.htm. We will not post any information on the web that will allow identification of individual patients or compromise the confidentiality of any participating laboratory. The purpose of these pages is to inform the clinical genetics community of CYTO2000 activities. Our web site will contain: inclusion criteria, participation forms, and timetables for on-going CYTO2000 projects; published results of CYTO2000 projects; and schedules and activities for upcoming CYTO2000 meetings.

The CYTO2000 is a cooperative venture. Although its immediate purpose is to provide answers to cytogenetic questions requiring pooled data from many laboratories, we hope that it also will serve as a model for studies in other disciplines when data from multiple sources is required to investigate important scientific questions.

\section{Reference}

1. Lejeune, J. Le mongolisme. Premier example d'aberrration autosomique humaine. Ann Genet 1959;i:41-49. National Center for Environmental Health Centers for Disease Control and Prevention Atlanta, Georgia

Paul S. Ing, PhD

Boys Town National Research Hospital Omaha, Nebraska

John A. Reidy, PhD

Division of Environmental Health Laboratory Sciences National Center for Environmental Health Centers for Disease Control and Prevention Atlanta, Georgia

Stuart Schwartz, PhD Center for Human Genetics Cleveland, Ohio

Gail H. Vance, MD

Medical and Molecular Genetics Indiana University School of Medicine Indianapolis, Indiana

Daniel L. Van Dyke, PhD Cytogenetics Laboratory Henry Ford Hospital Detroit, Michigan 